\title{
Assessment of Offshore Airport Artificial Island Construction Impact on Chlorophyll-a Using HJ-1A/B-CCD Satellite Data
}

\author{
Yu Tiaolan \\ Department of Transportation Management \\ Dalian Maritime University \\ Dalian, China \\ yutiaolan@dlmu.edu.cn
}

Wu Nuan

Department of Transportation Management

Dalian Maritime University

Dalian, China

wunuan@dlmu.edu.cn

\author{
Wang Nuo* \\ Department of Transportation Management \\ Dalian Maritime University \\ Dalian, China \\ wangnuodl@dlmu.edu.cn \\ * Corresponding author. Tel.: +86 41184726835.
}

\author{
Song Nanqi \\ Department of Transportation Management \\ Dalian Maritime University \\ Dalian, China \\ songnanqi@,dlmu.edu.cn
}

\begin{abstract}
It is possible to track and monitor the multitemporal marine pollution caused by large-scale reclamation engineering techniques by using remote-sensing techniques, which can overcome the spatial and temporal constraints of situ sampling. The concentration of chlorophyll-a is a widely used indicator of phytoplankton abundance and eutrophication in marine aquatic systems. It is important to monitor the dynamic variation in the chlorophyll-a concentration of large areas and take effective measurements in a timely manner. The impact of the construction of the Dalian offshore airport artificial island on water quality was investigated by using $\mathrm{HJ}-1 \mathrm{~A} / \mathrm{B}-\mathrm{CCD}$ satellite data regarding chlorophyll-a concentration at different time intervals. This study developed a satisfying regression model between the spectral reflection ratio and chlorophyll-a concentration based on $\mathrm{HJ}-1 \mathrm{~A} / \mathrm{B}-\mathrm{CCD}$ satellite data and field data gathered from the Dalian offshore artificial island in China. The retrieval precision of the linear retrieval model for the third and fourth bands of the HJ-1A/B-CCD sensor was enough to objectively reveal the marine chlorophyll-a concentration distribution and its variation characteristics during the construction. The feasibility and effectiveness of using remote sensing to track and monitor marine environments affected by large-scale offshore artificial island construction were proved.
\end{abstract}

Keywords- Remote sensing; Reclamation engineering; Chlorophyll-a; Marine environment; Pollution

\section{INTRODUCTION}

Large-scale marine reclamation is expected to pollute the surrounding marine environment and damage the halobios. The concentration of chlorophyll-a is a widely used indicator of phytoplankton abundance and eutrophication in marine aquatic systems. High concentrations of chlorophyll-a often indicate poor water quality, and low concentrations often suggest good conditions. The analysis of SS data gathered in situ using vessels has been commonly used to investigate states of marine pollution. However, this method is time-consuming and expensive, and has limitations when implemented over large areas. Thus, stations that are as small as possible and certain intervals of observation are used to monitor the marine environment. Clearly, field campaigns constitute an approximate method of monitoring results at certain stations to infer the general situation of an entire area. The results obtained using this method would not be credible over a wide range of temporal and spatial scales. Remotesensing techniques have recently garnered increasing attention because they can overcome the spatial and temporal limitations of traditional in-field observation.

There are some studies regarding the impacts of reclamation on the marine environment. Hemer et al. developed hydrodynamic models that consider the combined action of waves and tidal currents to calculate morphological evolution; the results of these models are in agreement with measured data ${ }^{[1]}$. Li et al. focused on water pollution and ecological damage during coastal engineering construction using water-quality models. On the west and south sides of the Bohai Sea ${ }^{[2]}$, Nie et al analysed the marine pollution caused by reclamations ${ }^{[3]}$ With respect to the use of remote-sensing technology to monitor the marine environment, Huang et al. investigated the impact of Hurricane Ivan on water quality in Pensacola Bay using Modis $250 \mathrm{~m}$ remote sensing data regarding chlorophyll-a concentrations at different time intervals before and after the hurricane event ${ }^{[4]}$; Song et al. 4implemented a hyperspectral quantitative model to monitor the chlorophyll-a concentrations of alga and suspended particles in Tai Lake, with spectral measurement and synchronous sampling performed using a portable spectroradiometer ${ }^{[5]}$; Yang et al.retrieved chlorophyll-a concentrations from Chaohu Lake with the three band model using HJ-1A/1B satellite hyperspectral data $^{[6]}$; Onderka et al. described how a Landsat ETM image was used to map the spatial patterns of suspended particulate matter in the Slovak portion of the Danube 
River based on a strong relationship between the Landsat near-infrared band (TM4) and field measurements ${ }^{[7]}$; Min et al. analysed the water quality of an estuary and bay in terms of chlorophyll-a and suspended sediment concentration using Modis satellite data ${ }^{[8]}$. Among these studies, some only focused on mathematical modeling, while others focused on the evolution of the natural environment; however, few used satellite data to detect the environmental changes caused by human activities such as large-scale offshore artificial island construction.

In this study, a method incorporating remote sensing was developed to monitor the marine pollution caused by the construction of the Dalian offshore airport artificial island, China, and a regression remote-sensing model was derived from the relation between in situ chlorophyll-a concentration and surface reflectance gathered from $\mathrm{HJ}$ imagery. The method and model were combined to determine the large-scale concentration of chlorophyll-a in the construction area.

\section{STUDY AREA AND DATA}

\section{A. Engineering Situation}

Jinzhou Bay is located in the eastern part of Bohai Bay, China, adjacent to the southwest end of the Liaodong Peninsula. The city of Dalian plans to build an offshore airport artificial island in Jinzhou Bay (Fig .1), which is connected to the mainland by a cross-sea bridge. The artificial island's area is $21 \mathrm{~km}^{2}$, which is $3 \mathrm{~km}$ away from the nearest shore. The water depth of the project area varies from $5 \mathrm{~m}$ to $8 \mathrm{~m}$, and the airport runway will require 60 million $\mathrm{m}^{3}$ of soil to be dredged and backfilled, in addition to 300 million $\mathrm{m}^{3}$ of backfilling earthwork. Largescale marine engineering must take measures to effectively monitor the environment surrounding the construction areas.

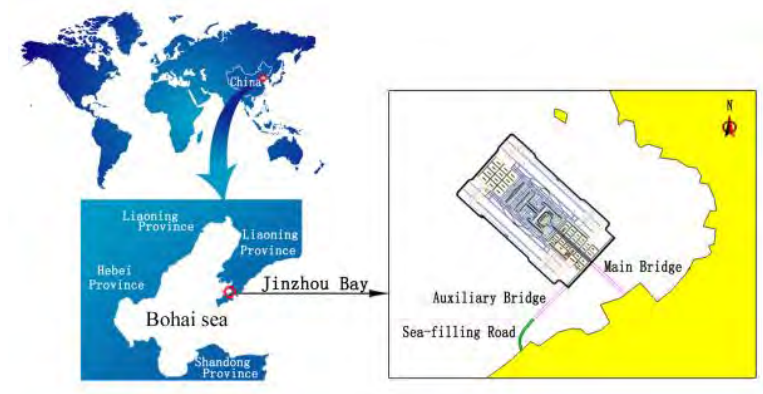

Figure 1. Location of Dalian offshore airport.

\section{B. In situ data collection}

According to the water flow distribution and possible pollution area in Jinzhou Bay, field surveys were performed at 12 sites around the offshore airport marked in Fig .2. The data were collected on November 16th, 2011 and August 14th, 2012. The corresponding geographical coordinates of the stations and the field results of chlorophyll-a concentration are shown in table 1. In accordance with China National Standards GB 17378.72007, spectrophotometry was used to measure the chlorophyll-a concentration.

\section{Satellite data}

Due to the relatively small study area, high spatial and temporal resolution is more suitable for monitoring variations in chlorophyll-a concentration caused by human activity. Thus, the Huanjing (HJ) images are the best choice.

$\mathrm{HJ}$ images acquired by the HJ-1A/1B satellites were obtained from the China Centre for Resource Satellite Data and Applications. The HJ-1A and HJ-1B satellites were launched on September 6, 2008 and passed the in-orbit-test review on February 19, 2009, after more than five months of in-orbit testing. The HJ-CCD sensor on board HJ-1A/1B has four bands in the visible and near-infrared spectrum, visible blue Band-1 (0.43-0.52 um), visible green Band-2 (0.52-0.60 um), visible red Band-3 (0.63-0.69 um) and near-infrared Band-4 (0.76-0.90 um), with a spatial resolution of $30 \mathrm{~m}$ and a revisiting time of two days.

As the peak of the chlorophyll-a spectral curve is between $450 \mathrm{~nm}$ and $650 \mathrm{~nm}^{[5]}$, the wavelength range of the HJ satellite data is suitable for the retrieval of chlorophyll-a concentration. To retain the synchronicity of the field data and satellite data, the dates that the HJ images were taken were November 14th 2011 and August 13 th 2012.

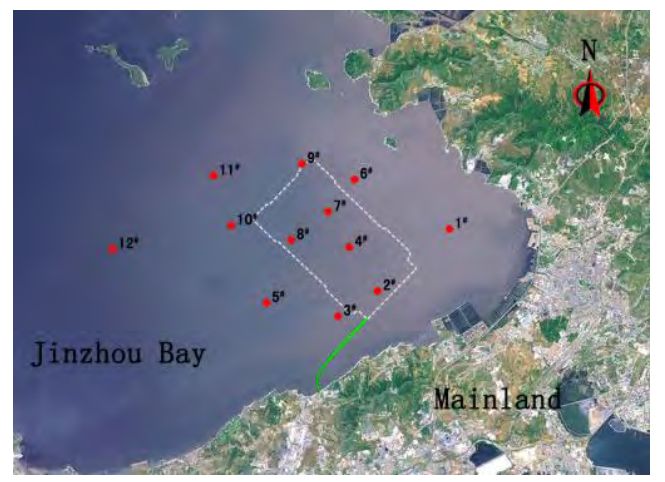

Figure 2. Location of observation sites in Dalian offshore airport

TABLE I. FIELD RESULTS FOR CHLOROPHYLL-A CONCENTRATION IN CONSTRUCTION AREA

\begin{tabular}{ccc}
\hline \multirow{2}{*}{ Station } & \multicolumn{2}{c}{ chlorophyll-a concentration $\left(\mathbf{u g ~ \mathbf { ~ } ^ { - \mathbf { 1 } } )}\right.$} \\
\cline { 2 - 3 } & November $\mathbf{1 6}^{\text {th }}, \mathbf{2 0 1 1}$ & August $\mathbf{1 4}^{\text {th }} \mathbf{2 0 1 2}$ \\
\hline $1 \#$ & 0.36 & $/$ \\
$2 \#$ & 0.36 & $/$ \\
$3 \#$ & 0.21 & $/$ \\
$4 \#$ & 0.28 & 0.74 \\
$5 \#$ & 0.25 & 0.72 \\
$6 \#$ & 0.31 & 0.68 \\
$7 \#$ & 0.30 & 0.62 \\
$8 \#$ & 0.25 & 0.89 \\
$9 \#$ & 0.25 & 0.57 \\
$10 \#$ & 0.33 & 0.63 \\
$11 \#$ & 0.51 & 0.55 \\
$12 \#$ & 0.43 &
\end{tabular}




\section{CHLOROPHYLL-A CONCENTRATION RETRIEVAL}

\section{A. Atmospheric correction}

In this paper, ENVI's Fast Line-of-sight Atmospheric Analysis of Spectral Hypercubes (FLAASH) was applied to correct the HJ images for atmospheric water vapour, oxygen, carbon dioxide, methane, ozone, and molecular and aerosol scattering using a MODTRAN 4+ radiation transfer code solution computed for each image and each pixel in the images.

According to the $\mathrm{HJ}-1 \mathrm{~A} / \mathrm{B}$ satellite absolute radiometric calibration coefficients published in China Resources Satellite Application Center in November 2011, the pixel value in the original image (DN) was converted to apparent radiance through Eq. (1)

$$
L_{\lambda}=D N / A+L_{0}
$$

where $L_{\lambda}$ denotes the measured apparent radiance; DN denotes the pixel value in the original image; A denotes the sensor's corresponding gain; and $L_{0}$ denotes sensor's corresponding bias. After conversion, the units of $L_{\lambda}$ were $\mathrm{W} \cdot \mathrm{m}^{-2} \cdot \mathrm{sr}^{-1} \cdot \mathrm{m}^{-1}$.

Upon implementing the radiometric calibration of the remotely sensed images, apparent radiance $L_{\lambda}$ can be converted to apparent reflectance $\rho_{\lambda}$ through Eq. (2) ${ }^{[9]}$.

$$
\rho_{\lambda}=\pi L_{\lambda} d^{2} / E_{\lambda} \cos \theta_{\lambda}
$$

In Eq. (2), $\rho_{\lambda}$ represents the apparent reflectance of the $\lambda$-band. $L_{\lambda}$ refers to the apparent radiance of the $\lambda$-band. $d$ is the earth-sun distance in astronomical units. $E_{\lambda}$ is the solar spectral irradiance of the $\lambda$-band in the upper atmosphere. $\theta_{\lambda}$ denotes the solar zenith angle.

Based on the theory of radiative transfer, assuming that an underlying surface is homogeneously Lambertian, the radiance received by a sensor above the atmosphere can be expressed as shown in Eq. (3).

$$
L_{\lambda}=L_{1}+[\rho /(1-s * \rho)] *\left[T F_{d} / \pi\right]
$$

where $L_{l}$ denotes the atmospheric radiance of zero land surface reflectance; $T$ refers to the transmittance from the land surface to the sensor; $s$ is the spherical albedo in the underlying atmosphere; $\rho$ is pixel reflectance; $F_{d}$ is the downward radiation flux density that reaches the land surface.

By combining Eq. (2) and (3), the pixel reflectance can be expressed using Eq. (4).

$$
\rho=\left[\pi \times\left(L_{\lambda}-L_{1}\right)\right] /\left[T F_{d}+\pi * s *\left(L_{\lambda}-L_{1}\right)\right]
$$

The values of $L_{1}, s$ and $T F_{d} / \pi$ can be obtained from the results of references [9].

\section{B. Chlorophyll-a retrieval}

The composition of case II water is complex, and the optical properties of case II water are more complex than those of case I water, due to the influence of phytoplankton, suspended particulate matter and chromophoric dissolved organic matter (CDOM). Therefore, the ratio method is widely used to retrieve chlorophyll-a concentrations. In this study, HJ-1A/B-CCD satellite data were selected. The basic form of Two-Band Ratio Model (TRM) is $R_{r s}\left(\lambda_{2}\right)$ ) $R_{r s}\left(\lambda_{2}\right)$, the band range of are $660 \sim 1000 \mathrm{~nm}$ and $700 \sim 780$ $\mathrm{nm}$, respectively. According to the constraints of the $\mathrm{TRM}^{[10]}$, the ratio of the fourth to the third band (Band4/Band-3) from HJ images was used as the independent variable in the TRM. The form of the retrieval model used, i.e., linear, logarithmic form or exponential, depended on the relationship between the aforementioned ratio and the measured data.

The independent variable was the ratio of the fourth to the third band from $\mathrm{HJ}-1 \mathrm{~A} / \mathrm{B}-\mathrm{CCD}$ satellite data, and the following models were constructed: quadratic model, exponential model, exponential model, power index mode and logarithmic model. Moreover, the models depended on the statistical relationship between the ratio and the in situ chlorophyll-a concentration; the corresponding model results are shown in Table 2.and $R s$ refers to Band-4/Band3, Cha refers to the concentration of chlorophyll-a. $R^{2}$ refers to the correlation coefficient, $R M S E$ refers to Root Mean Square Error, and RE is the general relative error.

This study used the RMSE and $R E$ to evaluate the advantages and disadvantages of the constructed models. The corresponding computation formulas are as follows: $R M S E=\sqrt{\sum\left(d_{m}-d_{p}\right)^{2} / n} \times 100 \% \quad, \quad R E=\left\lfloor\sum\left|d_{m}-d_{p}\right| / d_{m} \mid / n \times 100 \%\right.$ where $d_{m}, d_{p}$ and $n$ refer to measured data, simulation value and the number of sampling points, respectively. The optimal model is that with the minimum $R M S E$ value; if the RMSE values are similar among two or more models, the model with the highest $R^{2}$ value and lowest $R E$ value is optimal. The evaluation results shown in Table 2 indicate that the linear model was the best choice.

\section{Discussion of retrieval results}

The independent variable of the chlorophyll-a retrieval model for the Dalian offshore airport artificial island construction area was the ratio of band- 4 to band-3, and the optimal model was the linear model. The RMSE, $R^{2}$ $R E$ of the optical model were $19.68 \%, 0.7503,1.23 \%$, respectively. The concentration of chlorophyll-a according to the field ranged between 0.21 and $0.89 \mathrm{ug} \mathrm{L^{-1 }}$; retrieval results revealed concentrations ranging between 0.57 and $1.25 \mathrm{ug} \mathrm{L}^{-1}$. Five stations synchronous field data (August 14th 2012) were used to verify the retrieval model and the corresponding results; The associated RMSE (19.68\%) was below the upper limit $(30 \%)$ of retrieval precision in case II water. The verification showed that the retrieval model achieved great results by using band 4 and band 3 from the HJ CCD data. The retrieval of chlorophyll-a concentration distribution was described in Fig.3.

Comparing the field data with the retrieval results for chlorophyll-a concentration revealed the error between them. There were two reasons for this error. First, such strict control measurements of the pollutants were performed during the construction of the Dalian offshore airport artificial island that the area had little effect on the surrounding marine environment, and the chlorophyll-a concentration was also low. Second, weather was an important factor in the synchronisation of the field and satellite data. The satellite data were obtained one day or two days later than the field data, which reduced the precision of the retrieval. If the satellite data and the measured data's acquisition were completely synchronous, the precision of the retrieval would be improved.

\section{CONCLUSION}

Remote-sensing techniques are useful in monitoring and detecting marine pollution caused by human activities such as large-scale marine engineering projects. These techniques can overcome the spatial and temporal 
constraints of in situ sampling. Moreover, it is possible to monitor multi-temporal and large-scale construction areas. In this study, the chlorophyll-a concentration in Dalian offshore airport construction areas retrieved from HJ images and the corresponding retrieval results were compared to in-situ data. The results indicate that the logarithmic retrieval model built by the ratio of the third to fourth band from the HJ CCD sensor had high precision. Moreover, the retrieval results were able to reflect the distribution and variation characteristics of the chlorophyll-a concentration. According to the preliminary results obtained from the application of remote-sensing to monitor the variation in the concentration of chlorophyll-a, it is advantageous and effective to utilise the HJ satellites to detect the temporal and spatial distribution of chlorophyll-a variation in construction areas.

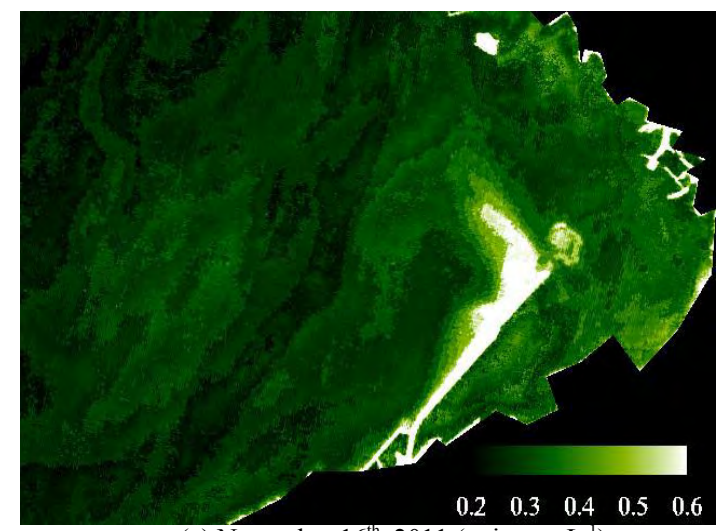

(a) November $16^{\text {th }}, 2011$ (units: ug L ${ }^{-1}$ )

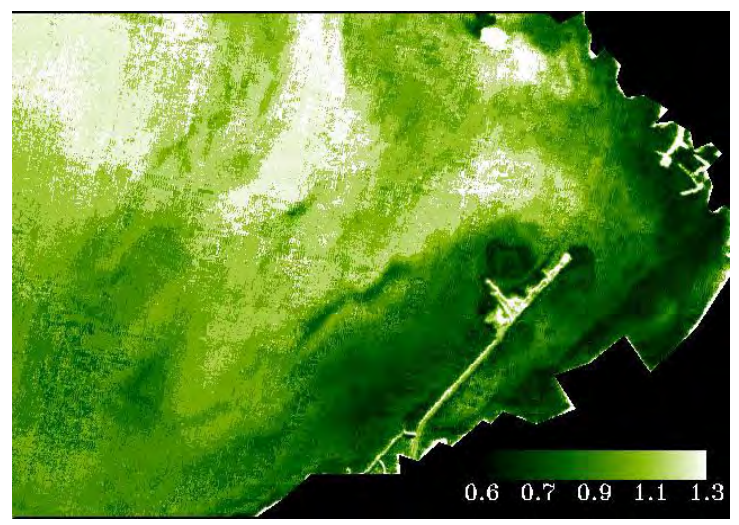

(b) August 14 $4^{\text {th }}, 2012$ (units: ug L ${ }^{-1}$ )

Figure 3. Distribution of chlorophyll-a concentration according to retrieval results
TABLE II. EVALUATION RESULTS OF RETRIEVAL MODELS

\begin{tabular}{|c|c|c|c|c|}
\hline Model types & Model equations & $R M S E(\%)$ & $R^{2}$ & $\begin{array}{c}R E \\
(\%)\end{array}$ \\
\hline Linear & $C h a=1259.7 R s+391.6$ & 19.68 & 0.7503 & 1.23 \\
\hline A quadratic & $\begin{array}{c}C h a=983.2 R s^{2}- \\
1879 R s+934.3\end{array}$ & 29.85 & 0.5710 & 2.01 \\
\hline Exponential & $\begin{array}{c}C h a=7973 \exp (- \\
23.1 R s)\end{array}$ & 30.01 & 0.4729 & 2.87 \\
\hline Power index & $C h a=2.87 R s^{(-7.124)}$ & 32.63 & 0.4396 & 3.95 \\
\hline Logarithmic & $\begin{array}{c}C h a=-219.7 \ln R s- \\
104.7\end{array}$ & 39.88 & 0.3642 & 3.17 \\
\hline
\end{tabular}

\section{ACKNOWLEDGMENT}

This research was supported by the National Natural Science Foundation of China (Grant No. 71372087), the Education Ministry Programming Foundation of China (Grant No. 12YJA630128), and the Liaoning science \& technology project (Grant No. 2012220008). The authors would like to thank colleagues from collaboration departments for assistance in data compilation, analysis, and interpretation.

\section{REFERENCES}

[1] M.A.Hemer, P.T.Harris, R.Coleman et al. Sediment mobility due to currents and waves in the Torres Strait-Gulf of Papua region. Continental Shelf Research, 2004, 24(19):2297-2316.

[2] T.H.Li, P.Han, Z.J.Zhao. Impact analysis of coastal engineering projects on mangrove wetland area change with remote sensing. China Ocean Engineering, 2008,2:347-358.

[3] H.T.Nie, J.H.Tao. Eco-Environment status of the Bohai Bay and the impact of coastal exploitation. Marine Science Bulletin, 2009,11(2):81-96

[4] W.R.Huang, D.Mukherjee, S.S.Chen. Assessment of Hurricane Ivan impact on chlorophyll-a in Pensacola Bay by MODIS $250 \mathrm{~m}$ remote sensing. Marine Pollution Bulletin, 2011, 62:490-498.

[5] Y.Song, X.D.Song, H.Jiang, et al. Quantitative Remote Sensing Retrieval for Algae in Inland Waters. Spectroscopy and Spectral Analysis, 2010,30(4):1075-1079.

[6] Y.Yang, Y.M.Li, Q.Wang, et al. Retrieval of chlorophyll-a concentration by Three-band model in Lake Chaohu. Journal of Lake Sciences, 2010, 22(4):495-503.

[7] M.Onderka, P.Pekárová. Retrieval of suspended particulate matter concentrations in the Danube River from Landsat ETM data.Science of The Total Environment, 2008, 397(1-3): 238-243.

[8] J.E.Min, J.H.Ryu. Monitoring of suspended sediment variation using Landsat and MODIS in the Saemangeum coastal area of Korea. Marine Pollution Bulletin, 2012,64(2), 382-390.

[9] S.Zheng, X.Zhao, H. Zhang, et al. Atmospheric correction on CCD data of HJ-1 satellite and analysis of its effect. Journal of Remote Sensing, 2011, 15(4):709-721.

[10] L.Zhou, L.H.Ma, H.T.Duan, , J G.J.iang, et al. Remote sensing retrieval for chlorophyll-a concentration in turbid case II waters (I): the optimal model. Journal of Infrared and Millimeter Waves, 2011, 30(6),531-537. 